\title{
Wo lag Ramath-Gilead?
}

Von Pfarrer Lic. Dr. Julius Boehmer in Raben (Mark).

Die Stadt Rama in Gilead wird auf den meisten Karten noch immer vergeblich gesucht, obwohl ihre Lage, sofern wir EusEBIUS' Onomastikon folgen, sehr klar ist. Hier wird nämlich zu Dtn 443 angegeben, da $\$$ sie, eine Priester- und Asylstadt, im Stamme Gad etwa I 5 römische Meilen westlich von Philadelphia (= Rabbat Ammon) gelegen habe; ferner zu I Reg 4 13, daß sie zur Zeit ein Dorf in Peräa am Jabbokflusse sei. Die erste Angabe trifft für das heutige Es-salt zu. Die zweite allerdings nicht, da diese Stadt nicht am Jabbokflusse gelegen ist. Aber der Irrtum EusEbs ist hier sehr begreiflich: denn da der Jabbok von Philadelphia aus zum Jordan, also nach dem Westen strebt und Es-salt tatsächlich im Westen von Philadelphia liegt, so konnte man leicht annehmen, daß der Jabbokfluß an Es-salt (= Ramath-Gilead) vorbei in den Jordan fließe - wenn. man nicht wußte oder übersah, daß der Jabbok von Philadelphia aus zunächst eine nördliche Richtung nimmt und erst dann nach Westen umlenkt, um mehrere Meilen nördlich von Ramath-Gilead vorbeizugehen.

Damit wäre nun alles klar, wenn nicht neuerdings gegen diese so plausible Identifizierung Einwände erhoben wären. Zunächst daß Es-salt einem alten Gedor encspreche. So z. B. GUTHE im Bibelwörterbuch S. 537. Dieses Gedor freilich muß dann GuTHE S. 200 weiter auf Gadara zurückschieben. Unter diesem Titel. ist (abgesehen von dem Gadara im JarmukTal in der Nähe des Sees von Genezareth, dem heutigen Mukēs) gesagt: „Die feste Hauptstadt von Perả, aus' der die aufständischen Juden vor Vespasian 68 n. Chr. nach Bethennabris (= Beth Nimra) flohen.

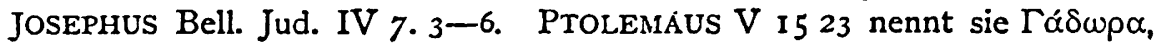
die judischen Gelehrten $\Gamma \varepsilon \delta \omega \rho$. Sie entspricht dem heutigen Es-salt, dessen Quelle noch den Namen 'ain ǵadûr fuhrt." Wer JOSEPHUS' Erzàhlung mit Aufmerksamkeit verfolgt, kann nicht zweifeln, daß das von ihm a. a. O. genannte Gadara allerdings ein anderes sein muß als das des Jarmuktals, da ganz deutlich Bethennabris oder Nimra in der Nähe Gadaras liegt. 
Wenn nun Nimra im Stamme Gad auf der Südseite des Wadi-Nimrin, an dessen oberen Eingang (von hier bis zum Beginn des Ghor sagt man: Wadiesch-scha'ib) auch Es-salt liegt, zu suchen ist, und zwar an der Stelle, wo der Wadi das Ghor erreicht, uber $1000 \mathrm{~m}$ tiefer als Es-salt gelegen, auf der Straße zum Jordan und nach Jericho hin, das von Es-salt aus in einer Tagereise $z u$ erreichen ist; so ist die Situation ganz klar und auber Essalt kann fur Gadara in der Tat keine andere Stadt hier in Betracht kommen. Allerdings will SCHÜRER diese ldentifikation nicht gelten lassen. Er macht (Gesch. des juid. Volk. II 125) geltend, daß mit dem Ausdruck

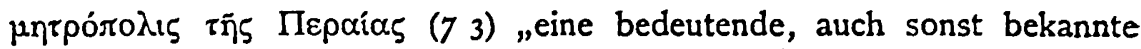
Stadt" bezeichnet sein müsse, und stellt fest, daß der Sinn jenes Ausdrucks nicht $z u$ ermitteln sei. Allein mit dieser Feststellung ist eigentlich auch die Bedeutung und sonstige Bekanntheit der Stadt ein wenig in Frage gestellt, allerdings auch das Recht zu der irrefuhrenden Übersetzung mit „Hauptstadt", deren sich nicht bloß GUTHE bedient, umgestoßen. Etwa „Vorort", „Vorstadt" im alten, von dem modernen abweichenden Sinn durfte annähernd das Richtige sein, wobei die nähere Bestimmung, ob „Vorort einer Festgemeinschaft zur Feier periodischer Festspiele" (so ECKHEL nach SCHÚRER a. a. O.) oder was sonst gemeint sei, dahingestellt bleiben muß. Wissen wir von Gadara-Mukēs nichts anzugeben, was jenen Ausdruck verständlich macht, so kann er sicherlich auch von jeder anderen Stadt gebraucht sein, die sonst in den $\mathrm{Zu}$ sammenhang pabt. Gadara-Mukēs aber paßt bestimmt nicht in den Zusammenhang. Denn dab die Flüchtlinge das ganze Ghor vom Jarmuk bis fast an das Tote Meer sollten durcheilt haben, daß sie diesen weglosen Weg genommen und ihn so weit unbehelligt hätten zurücklegen konnen, das ist einfach unglaubhaft - ebenso da $\mathrm{b}$ eine Heeres-Abteilung von 3000 römischen Fußsoldaten und 500 Reitern zur Verfolgung einen derartig weiten und schlechten Weg in den Kauf genommen hätte. ${ }^{x}$

Dagegen stimmt alles, wenn Gadara dem heutigen Es-salt entspricht: dann war die Flucht uber Nimra die einzig mögliche, und so beschwerlich sie sein mochte, so fuhrte sie doch an den Jordan und uber den Jordan nach Jericho, in Sicherheit.

Nach allem also werden Schlatter, Guthe, BuHL und die ubrigen Vertreter der Süd-Gadara-Hypothese im Rechte sein. Es hat keinerlei Schwierigkeit, zwei Orte desselben Namens im Ostjordanlande anzu-nehmen: wieviele Ortsnamen in Palästina wiederholen sich ein oder mehrere

× Andere Gegeninstanzen macht SchLATrer, Topographie S. 44 f. geltend. 
Male! ₹ Zudem ist für beide Gadara noch heute der alte Name erhalten: im Osten von Nord-Gadara für die Grabhöhlen in der Form von gedâr (gedûr), an der Quelle von Süd-Gadara, wie schon erwähnt, gadûr. Ebensowenig hat es Schwierigkeiten, einen doppelten Namen für denselben Ort festzustellen: Gadara ist eine andere Bezeichnung für das alte Ramath-Gilead. Hellenistisch kann sie schwerlich genannt werden, da der Name vielmehr sprachlich auf semitischen Ursprung zuruickweist. Aber die Annahme liegt nahe genug, daß sie in den Syrerkriegen zerstört wurde und lange in Trümmern gelegen hat, bis sie viel spater unter anderem Namen wieder aus den Trümmern erstand. Der Name Gadara aber legte sich. fur eine feste Stadt nahe genug: denn 7 , doch wohl aus einem ursprünglichen בַית־גָדר (Jos 1213 - I Chron 251) verkürzt wie das genannte Nimra (Num 323 ) aus Beth-Nimra (Num 3236 Jos 13 27), hängt wohl mit Mauer גָּ zusammen, und Ableitungen von diesem Stamm als Ortsbezeichnungen sind keineswegs selten, z. B. גְרו I Chron 4 4 39, ${ }^{2}$ דגדרוות Jos Ios 15 41. Von dieser Seite her also darf es als festgestellt gelten, da $\mathrm{D}$ der Gleichsetzung von Ramath-Gilead mit einem späteren Gadara und dem heutigen Es-salt nichts im Wege steht.

Ein zweiter Einwand gegen die Gleichsetzung von Ramath-Gilead mit Es-salt wird von Guthe a. a. O. der Stelle I Reg 4 I3 entnommen: diese erfordere eine viel nördlichere Lage als Es-salt, weil der Statthalter in Ramath-Gilead den Argob in Basan unter sich und noch zwei andere Statthalter v. 14 is offenbar nach Süden hin neben sich habe. Wer nun die in Betracht kommenden Stellen im Zusammenhang pruft, wird zu I Reg 47-19 folgende Beobachtungen machen. Laut der Ankundigung von v. 7 sollen die zwölf Ökonomie-Vögte Salomos, die er uber ganz Israel verteilt hatte, aufgezahlt werden. In der Tat werden v. 8-19 ihrer zwölf aufgezahlt. Und zwar v. 8-12 funf im Westjordanlande, dann v. 1314 zwei im Ostjordanlande, sodann v. $15-18$ vier wieder im Westjordanlande und v. 19 wieder einer im Ostjordanlande: v. 8-12 ist das westjordanische Gebiet vom Süden her bis an den Nordrand der JesreelEbene, v. 1314 das um Ramath-Gilead und Mahanaim gruppierte Gebiet des Ostjordanlandes, v. 15-17 die Gebiete der Stämme im Norden der Jesreel-Ebene, v. is das Gebiet Benjamins und v. 19 wieder das Ostjordan-

- Daher ist es auch nicht angāngig, mit Gutres Bibelwörterbuch 472 die Er-

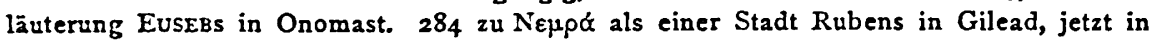
Basan gelegen, kurzerhand für falsch zu erklären: dies Nimra kann sehr wohl neben dem obengenannten bestanden habed. Allerdings fehlt der Name in unserm Richterbuch.

2 Ob hier, wie z. B. Schlatrer (Topographie S. 50) annimmt, unser Gadara gemeint ist, muf nach dem Gesagten als sehr zweifelhaft erscheinen. 
land unter dem Namen „Land Gilead" genannt. Schon diese Reihenfolge in der Aufzahlung ist höchst merkwurdig. Fast noch merkwurdiger ist folgendes: die v. 8-11 13 genannten Beamten haben keinen Eigennamen, sondern gehen unter der Firma: ,Sohn des ..."; die übrigen v. 12 14-19 werden mit Eigennamen und Vaternamen bezeichnet, nur dem v. 15 erwahnten Vogt fehlt der Vatername, wiewohl er Schwiegersohn des Konigs zu sein gewurdigt ward.

Was ist aus dem allen, aus der zwiefachen Ungleichmäbigkeit zu schließen? Wir beachten hier nur das, was fur unsere Frage in Betracht kommt. Die Liste v. 8-19 ist nicht einheitlich, sondern zusammengesetzt, um nicht zu sagen: zusammengestumpert. Und zwar hóchst eigentümlich, in der Weise, daß - so scheint es - dem Verfasser in der Tat amtliches Material vorlag, und er dieses möglichst peinlich genau oder wahllos-willkürlich verwertete. Die eine Liste, vielleicht oder wahrscheinlich: Vorschlagliste, hảtte die Personennamen ausgelassen und nur den Vaternamen notiert v. 8-13. Da hat dann einer, dem die Benennung "Sohn Ahiluds" undeutlich zu sein schien, vielleicht ein Revisor, in v. 13 den Personennamen Baana eingeschaltet. Die andere Liste, v. 14-19 wiedergegeben, hatte die Personennamen mit Vaternamen verzeichnet und glaubte v. 15 wohl, fur einen Schwiegersohn des Königs bedürfe es der Hinzufügung des Vaternamens nicht: dies gilt auch für den Fall, daß dies nicht der Priester Zadok sein sollte, dessen Sohn nach II Sam I5 $27361717 \mathrm{ff}$. allerdings Ahimaaz hieß. Die beiden Listen waren nicht ganz ohne innere Beziehung zueinander: der Name Baana, Name eines Vogts, kam in beiden vor, Geber ist in der einen Vatername, in der anderen Vogtname und zwar hier beides an letzter Stelle, während, daß Abinadab als Vatername in der einen und Ahinadab als Vogtname in der zweiten vorkommt, wie zufallig sein könnte.

Aus diesem Tatbestand ist zu erschließen:

I. Beide Listen sind Bruchstücke, Teile der Gesamt-Liste: 8-13 14-19.

2. Der Verfasser hat sie wohl in ausfuhrlicherer Gestalt, vielleicht sogar in ihrem gesamten Umfang vor. sich gehabt, und da sie nicht recht zusammenstimmten - es waren ja beides nur Vorschlagslisten - sie bearbeitet und zusammengearbeitet, so gut es gehen wollte.

Daraus. erklärt sich die so oft monierte stilistische Schwerfälligkeit in der Beschreibung der Amtsbezirke, die so regellose Aufeinanderfolge der Bezirke. Die vorhandenen Verschiedenheiten sind, wie gezeigt, auch 
wieder in gewissem Sinn einheitlich geartet. Doch nicht ganz: eine wirklich einheitliche, in sich selbst zusammenstimmende Liste hat der Verfasser nicht zustande gebracht, nicht zustande bringen wollen noch konnen. Wer v. $13 \mathrm{I}_{4}$ mit v. 19 vergleicht, sieht das gleich. Denn wenn laut v. 13 der Landstrich Argob in Basan dem Vogt in Ramath-Gllead zugeteilt war, wie kann laut v. 19 der Vogt von Gilead auch die Landschaft Basan verwalten? Es ist gewiß nicht zufällig, daß fur Basan einmal (v. 13) ein Sohn Gebers, ein andermal (v. 19) ein Geber als Vogt genannt wird: hier wird beide Male dieselbe Personlichkeit gemeint sein. Die Vögte Salomos, ihre Existenz und die Abgrenzung ihrer Bezirke in allen Ehren: aber ihre schriftliche Aufnahme scheint nicht geringe Schwierigkeiten gehabt zu haben, und die werden darauf zuruckzufuhren sein, daß die Einteilung des Landes in zwolf Bezirke sich nicht so eınfach vollzog, wie in einer Zeit, wo man Karten und Listen und Verzeichnisse aller Art schriftlich vor. sich hat und zugrunde legen kann. Was wir also v. 8-19 vor uns haben, ist nicht das amtliche Verzeichnis, sondern hòchstens Bearbeitung noch nicht abgeschlossenen, daher mangelhaften amtlichen Materials. Das eine Stück v. 8-13 enthielt sechs Bezirke.
I. Ephraim.
2. West-Juda.
3. Sud-Juda.
4. Strandebene von Jafa
5. Ebene Jesreel.
6. Bezirk von

bis zum Karmel

Ramath-Gilead.

Das andere Stuck v. $14-19$ umfaßte wieder sechs Bezirke:
7. Bezirk von Mahanaim.
8. Naphtali
9. Asser.
10. Isaschar.
II. Benjamin.
12. Gilead (ein- schließlich Basan).

Die Anordnung von 1.-6. läßt eigentlich nichts $\mathrm{zu}$ wüschen ubrig und der Anschluß von 7. ist auch tadellos. Daß man nach 7. Mahanaim Naphtali, Asser, Isaschar in 8.-10. folgen läßt, ist nicht minder der natürlichen Ordnung entsprechend, wàhrend allerdings II. Benjamin auf I0. Isaschar etwas unerwartet folgt, von 12. ganz zu geschweigen. Aber im zweiten Stuck v. 14-19 überhaupt stoßen sich 7. und 12. schon an sich, 12. außerdem noch mit 6. Eine weitere Aufklarung als die oben angedeutète $z u$ geben, ist hier nicht der Ort. ${ }^{3}$ Es genigt auch für unsern Zweck, festzustellen, daß.zum Verstandnis von 6. vorerst 7. und

I Nur erinnert sei daran, dab LXX das Obige insofern bestätigen, als hier 1. zum ersten Vaternamen in $r .8$ der Sohnesname mit Bez̀v genannt ist; 2 . am Schluß von v. ${ }_{28} \mathrm{Juda}$ hinzugefügt wird und dadurch 3. 13 Bezirke herauskommen; endlich 4. die Reibenfolge von 10.-13: hier ist: Benjamin, Gad (statt Gilead), Juda, Isaschar. 
erst recht 12. auber Betracht $\mathrm{zu}$ lassen ist, und dals man also nicht mit GurHe a. a. O. sagen kann, der Statthaiter in Ramath-Gilead habe noch zwéi andere Statthalter, offenbar nach Süden hin, neben sich gehabt.

Es ist an sich schon wenig wahrscheinlich, dalj Ramath-Gilead wo anders als in Gilead gelegen haben sollte. Da der Stadtname alt ist, so wird auch anzunehmen sein, dab hier Gilead im alten, also béschränkteren Sinn gebraucht wird, also die Landschaft östlich vom Jordan mit Ausschluß von Basan bezeichnet. So allein stimmt es auch mit der Ausdrucksweise v. 13, zu der die ganz analoge in v. $10 \mathrm{zu}$ vergleichen ist, daß nämlich der Argob in Basan als au@erhalb Ramath-Gileads liegend, noch zum Gebiet Gilead hinzukommend zu denken ist. Nun ist außerdem der Name Gilead, der als Landschaftsname einen bald engeren, bald weiteren Sinn hat, ursprünglich Gebirgs- oder Bergname, und wohl nach diesem die Stadt wie die Landschaft benannt. Dann aber ist erst recht nichts natürlicher als daß Ramath-Gilead mit Es-salt, das am Südful des Gebirges Gilead (heute Dschil'ad) liegt, zusammenfällt. Nebenbei gesagt, sieht es auch recht unwahrscheinlich aus, daß Salomo einen Vogt so weit an die außerste Grenze seines Reichs, bis ins Argob hinein, vorgeschoben haben sollte. ${ }^{x}$

\section{„Von Dan bis Berseba“.}

Von Pfarrer Lic. Dr. Julius Boehmer in Raben (Mark).

Wenn der Israelit alter Zeit das Gebiet seines Volkes überschaute und zum Ausdruck bringen wollte, was der deutsche Patriot vergangener Jahrhunderte in den Worten des Liedes meinte:

\section{Von der Maas bis an den Memel, \\ Von der Etsch bis an den Belt -}

I BUHL, Geographie S. 26If. sagt: „,ist die Angabe des Eusebius richtig (dab Ramot-Gilead 15 romische Meilen westlich von Philadelphia am Flusse Jabboq lag), so ist es eine Unmöglichkeit, Ramot nördlich vom Jabboq zu suchen. Unter den vorgeschlagenen Zusammenstellungen scheint die Kombination von Ramot mit der Ruine El-gal'aud ungefähr $5 \mathrm{~km}$ südlich vom Jabboq die größte Wabrscheinlichkeit für sich $z \mathfrak{u}$ haben. Hierfür spricht teils der Name, der auf ein altes Giléad zurückweist, teils die Lage, indem der Jabboq von hier aus sichtbar ist, und die Ebene am Fuße des Berges einen Kampf der Streitwagen (I Reg $22_{34} \mathrm{f}_{\text {. }}$ ) möglıch macht. Vielleicht ist auch die von Hosea erwähnte Stadt Gile'ad (Hos 68 vgl. Ri 12, LXX Cod. Al. und Lag.) mit Ramot-Gilead identisch und hier zu suchen.... Die von mehreren vorgeschlagene Kombination des Ortes Es-Salt mit dem Giléaditischen Ramot ist nicht wahrscheinlich, weil die Berge und Täler der Umgegend den Gebrauch der Streitwagen 